\title{
Verification of an Audio Protocol with Bus Collision Using UPPAAL ${ }^{\star}$
}

\author{
Johan Bengtsson ${ }^{2} \quad$ W.O.David Griffioen ${ }^{3,4} \quad$ Kåre J. Kristoffersen ${ }^{1}$ \\ Kim G. Larsen ${ }^{1} \quad$ Fredrik Larsson ${ }^{2} \quad$ Paul Pettersson ${ }^{2} \quad$ Wang $Y^{2}$ \\ 1 BRICS $^{\ddagger}$, Aalborg University, Denmark. E-mail: \{jelling,kgl\}@iesd.auc.dk \\ ${ }^{2}$ Department of Computer Systems, Uppsala University, Sweden. \\ E-mail: $\{$ johanb,fredrikl,paupet,yi\}@docs.uu.se \\ ${ }^{3}$ CWI, Amsterdam, The Netherlands. E-mail: griffioe@cwi.nl \\ 4 Computing Science Institute, University of Nijmegen, The Netherlands.
}

\begin{abstract}
In this paper we apply the tool UPPAAL ${ }^{1}$ to an automatic analysis of a version of the Philips Audio Control Protocol with two senders and bus collision handling. This case study is significantly larger than the real-time/hybrid systems previously analysed by automatic tools. During the case study the tool UPPAal was extended with a new feature, committed locations, allowing efficient modelling of broadcast communication.
\end{abstract}

\section{Introduction}

During the last few years a number of tools for automatic verification of hybrid and real-time systems have emerged [DY95, HHWT95, BLL +95 , HRP94]. These tools have by now reached a state, where they are mature enough for application on realistic case-studies; a claim we hope to substantiate in this paper.

We present an application of our tool UPPAAL to an automatic analysis of a version of the Philips Audio Control Protocol with two senders and the consequently caused problem of bus collision. The case study is comprehensive compared with previous verification efforts of real-time and hybrid systems, e.g. the node-space is $10^{3}$ times larger than the case with only one sender [BPV94, HWT95, DY95, LPY95]. Also, the number of clocks, variables and channels has increased considerably. The bus collision version studied in this paper has previously been verified in [Gri94] without tool support.

UPPAAL is a tool for automatic verification of safety and bounded liveness properties of networks of timed automata and certain hybrid automata. UPPAAL

\footnotetext{
* This work has been supported by the European Communities (under CONCUR2 and REACT), NUTEK (Swedish Board for Technical Development) TFR (Swedish Technical Research Council) and Netherlands Organization for Scientific Research (NWO) under contract SION 612-316-125.

₹ Basic Research in Computer Science, Centre of the Danish National Research Foundation.

${ }^{1}$ The current version of UPPAAL is available on the World Wide Web via the UPPAAL home page http://ww.docs.uu.se/docs/rtmv/uppaal.
} 
contains a number of features including a graphical interface and automatic generation of diagnostic traces, and applies a combination of on-the-fly statespace examination together with efficient constraint solving techniques [YPD94,

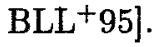

In modelling the Audio Protocol with bus collision it turned out to be convenient in certain situations to apply broadcast communication. An extension of UPPAAL with so-called committed locations allows broadcasts to be modelled as atomic sequences of two-process synchronizations, and yields in addition performance improvements.

The verification of Philips Audio Protocol with Bus Collision was carried out using the extended version of UPPAAL installed on a SGI ONYX machine. As results we have verified the correctness of the protocol for an error tolerance of $5 \%$ on the timing, demonstrated that correctness fails if the error tolerance is increased to $6 \%$, and analysed an incorrect version of the protocol which is actually implemented by Philips.

\section{The Committed UPPAaL model}

The basis of the UPPAAL model for real-time systems is networks of timed automata [AD90] with data variables [YPD94]. However, to meet requirements arising from various case studies, the UPPAAL model has been extended with various new features such as urgent transitions [BLL $\left.{ }^{+} 95\right]$ etc. The present case study indicates that we need to further extend the UPPAAL model with committed locations to model behaviours such as atomic broadcasting in real-time systems. Our experiences with UPPAAL show that the notion of committed locations introduced in UPPAAL is not only useful in modelling but also yields significant improvements in performance.

We assume that a real-time system consists of a fixed number of sequential processes communicating with each other via channels. We further assume that each communication synchronizes two processes as in CCS. Broadcasting communication can be implemented in such systems by repeatedly sending the same message to all the receivers. To ensure atomicity of such 'broadcast' sequences, we mark the intermediate locations of the sender as so-called committed locations which are to be left immediately.

An Example. To introduce the notion of committed locations in timed automata, consider the scenario shown in Figure 1: A sender $S$ is to broadcast a message $m$ to two receivers $R_{1}$ and $R_{2}$. As this requires synchronization between three processes this can not directly be expressed in UPPAAL where synchronization, as in CCS, is between two processes based on complementarity of actions. However, as an initial attempt we may model the broadcast as a sequence of two two-process synchronizations, where first $S$ synchronizes with $R_{1}$ on $m_{1}$ and then with $R_{2}$ on $m_{2}$. However, this is not an accurate modelling as the intended atomicity of the broadcast is not preserved (i.e. other processes may interfere during the 'broadcast' sequence). To ensure atomicity, we mark the intermediate location $\mathrm{S}_{2}$ of the sender $\mathrm{S}$ as a so-called committed location (indicated by the 


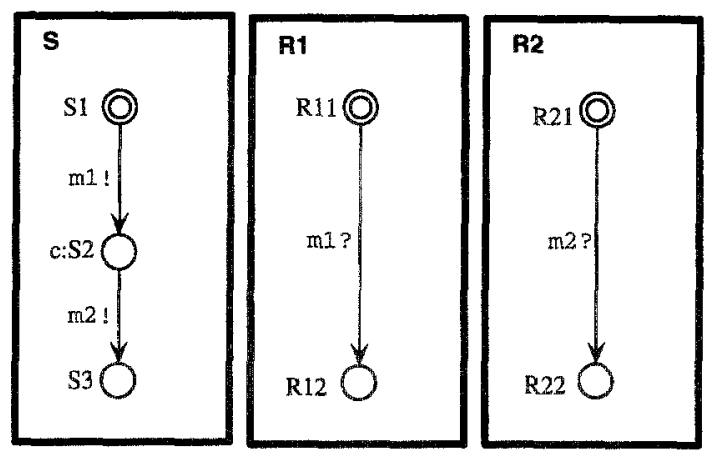

Fig. 1. Broadcasting Communication and Committed Locations.

c:-prefix). The atomicity of the action sequence $m_{1} ! m_{2} !$ is now achieved by insisting that a committed location must be left immediately! This behaviour is quite similar to what has been called "urgent transitions" [HHWT95, DY95, BLL+95] which insists that the next transition taken must be an action (and not a delay). The precise semantics of committed locations will be formalized in the transition rules for networks of timed automata with data variables in the following.

Preliminaries. We assume a finite set of clock variables $C$ ranged over by $x, y, z$ and a finite set of data variables $V$ ranged over by $i, j, k$. We use $G(C, V)$ to stand for the set of formulas ranged over by $g$, generated by the following syntax: $g::=a \mid g \wedge g$, where $a$ is a constraint of the form: $x \sim n$ or $i \sim n$ for $x \in C$, $i \in V, \sim \in\{\leq, \geq,=\}$ and $n$ being a natural number. We shall call elements of $G(C, V)$ guards. To manipulate clock and data variables, we use reset-set of the form: $\bar{w}:=\bar{e}$ which is a set of assignment-operations in the form $w:=e$ where $w$ is a clock or data variable and $e$ is an expression. A reset-set is a proper reset-set when the variables are assinged a value at most once, we use $R$ to denote the set of all proper reset-sets. A reset-operation on a clock variable should be in the form $x:=n$ where $n$ is a natural number and a reset-operation on an integer variable should be in the form: $i:=c * i+c^{\prime}$ where $c, c^{\prime}$ are integer constants. We assume that processes synchronize with each other via channels. Let $A$ be a set of channel names with a subset $U$ of urgent channels on which processes should synchronize whenever possible. We use $\mathcal{A}=\{\alpha$ ? $\mid \alpha \in A\} \cup\{\alpha ! \mid \alpha \in A\} \cup\{\tau\}$ to denote the set of actions that processes can perform to synchronize with each other, where $\tau$ is a distinct symbol representing internal actions. We use name $(a)$ to denote the channel name of $a$, defined by name $(\alpha ?)=$ name $(\alpha !)=\alpha$.

The UPPaAl Model with Committed Locations. An automaton $A$ over actions $A$, clock variables $C$ and data variables $V$ is a tuple $\left\langle N, l_{0}, E, N_{C}\right\rangle$ where $N$ is a finite set of locations (control-locations) with a subset $N_{C} \subseteq N$ being the set of committed locations, $l_{0}$ is the initial location, and $E \subseteq N \times G(C, V) \times A \times$ $R \times N$ corresponds to the set of edges. To model urgency, we require that the guard of an edge with an urgent action should always be th, i.e. if name $(a) \in U$ and $\left\langle l, g, a, r, l^{\prime}\right\rangle \in E$ then $g \equiv t$. 
In the case, $\left\langle l, g, a, r, l^{\prime}\right\rangle \in E$ we shall write, $l \stackrel{g, a, r}{\longrightarrow} l^{\prime}$ which represents a transition from the location $l$ to the location $l^{\prime}$ with guard $g$ (also called the enabling condition of the edge), action $a$ to be performed and a set of reset-operations $r$ to update the variables. Also, we shall write $\mathcal{C}(l)$ whenever $l \in N_{C}$.

To model networks of processes, we introduce a CCS-like parallel composition operator for automata. Assume that $A_{1} \ldots A_{n}$ are automata. We use $\bar{A}$ to denote their parallel composition. The intuitive meaning of $\bar{A}$ is similar to the CCS parallel composition of $A_{1} \ldots A_{n}$ with all actions being restricted, that is, $\bar{A}=$ $\left(A_{1}|\ldots| A_{n}\right) \backslash \mathcal{A}$. Thus only synchronization between the components $A_{i}$ is possible. We shall call $\bar{A}$ a network of automata. We simply view $\bar{A}$ as a vector and use $A_{i}$ to denote its $i$ th component.

Informally, a process modelled by an automaton starts at location $l_{0}$ with all its variables initialized to 0 . The values of the clocks increase synchronously with time at location $l$. At any time, the process can change location by following an edge $l \stackrel{g, a, r}{\longrightarrow} l^{\prime}$ provided the current values of the variables satisfy the enabling condition $g$. With this transition, the variables are updated by $r$.

A variable assignment is a mapping which maps clock variables $C$ to the nonnegative reals and data variables $V$ to integers. For a variable assignment $v$ and a delay $d, v \oplus d$ denotes the variable assignment such that $(v \oplus d)(x)=v(x)+d$ for any clock variable $x$ and $(v \oplus d)(i)=v(i)$ for any integer variable $i$. This definition of $\oplus$ reflects that all clocks operate with the same speed and that data variables are time-insensitive. For a reset-operation $r$ (a set of assignment-operations), we use $r(v)$ to denote the variable assignment $v^{\prime}$ with $v^{\prime}(w)=\operatorname{val}(e, v)$ whenever $w:=e \in r$ and $v^{\prime}\left(w^{\prime}\right)=v\left(w^{\prime}\right)$ otherwise, where $\operatorname{val}(e, v)$ denotes the value of $e$ in $v$. Given a guard $g \in G(C, V)$ and a variable assignment $v, g(v)$ is a boolean value describing whether $g$ is satisfied by $v$ or not.

A control vector $\bar{l}$ of a network $\bar{A}$ is a vector of locations where $l_{i}$ is a location of $A_{i}$. We shall write $\bar{l}\left[l_{i}^{\prime} / l_{i}\right]$ to denote the vector where the $i$ th element $l_{i}$ of $\bar{l}$ is replaced by $l_{i}^{\prime}$.

A state of a network $\bar{A}$ is a configuration $\langle\bar{l}, v\rangle$ where $\bar{l}$ is a control vector of $\bar{A}$ and $v$ is a variable assignment. The initial state of $\bar{A}$ is $\left\langle\bar{l}_{0}, v_{0}\right\rangle$ where $\bar{l}_{0}$ is the initial control vector whose elements are the initial locations of $A_{i}$ 's and $v_{0}$ is the initial variable assignment that maps all variables to 0 .

To model progress properties, we use the following notion of maximal delay:

$$
\mathrm{MD}(l, v)= \begin{cases}0 & \text { if } \mathcal{C}(l) \\ \max \left\{d \mid l \stackrel{g, a, r}{\longrightarrow} l^{\prime} \text { and } g(v \oplus d)\right\} & \text { otherwise }\end{cases}
$$

So if $l$ is a committed location, there will be no delay at $l$. We extend the notion of maximal delay to networks of automata such that synchronization on urgent channels happens immediately:

$\operatorname{MD}(\bar{l}, v)= \begin{cases}0 & \text { if } \exists \alpha \in U, i \neq j, l_{i}, l_{j} \in \bar{l}: l_{i} \stackrel{\alpha ?, r_{i}}{\longrightarrow} \& l_{j} \stackrel{\alpha !, r_{j}}{\longrightarrow} \\ \min \{\operatorname{MD}(l, v) \mid l \in \bar{l}\} & \text { otherwise }\end{cases}$

The semantics of a network of automata $\bar{A}$ is given in terms of a transition 
system with the set of states being the set of configurations and the transition relation defined as follows:

$-\langle\vec{l}, v\rangle \sim\left\langle\bar{l}\left[l_{i}^{\prime} / l_{i}\right], r_{i}(v)\right\rangle$ if there exist $l_{i} \in \bar{l}, g_{i}, r_{i}$ such that $l_{i} \stackrel{g_{i}, r, r_{i}}{\rightarrow} l_{i}^{\prime}, g_{i}(v)$, and for all $k$ if $\mathcal{C}\left(l_{k}\right)$ then $k=i$.

$-\langle\bar{l}, v\rangle \sim\left\langle\bar{l}\left[l_{i}^{\prime} / l_{i}, l_{j}^{\prime} / l_{j}\right],\left(r_{i} \cup r_{j}\right)(v)\right\rangle$ if there exist $l_{i}, l_{j} \in \bar{l}, g_{i}, g_{j}, \alpha, r_{i}$ and $r_{j}$ such that $i \neq j, l_{i} \stackrel{g_{i}, \alpha !, r_{i}}{\longrightarrow} l_{i}^{\prime}, l_{j} \stackrel{g_{j}, \alpha ?, r_{j}}{\longrightarrow} l_{j}^{\prime}, g_{i}(v), g_{j}(v), r_{i} \cup r_{j} \in R$ and for all $k$ if $\mathcal{C}\left(l_{k}\right)$ then $k=i$ or $k=j$.

$-\langle\bar{l}, v\rangle \sim\langle\bar{l}, v \oplus d\rangle$ if $d \leq \mathrm{MD}(\vec{l}, v)$

Thus, if a state $\langle\bar{l}, v\rangle$ contains a committed location no delays can take place. Moreover, any component with committed location must participate in the next (action-) transition.

\section{The Committed UPPAAL Implementation}

In the following, we present the notion of committed locations in terms of the UPPAAL model and its implementation in UPPAAL. In the current version $\left[\mathrm{BLL}^{+} 95\right]$, UPPAAL is able to check for invariance properties, $\forall \square \beta$, and reachability properties, $\exists \diamond \beta$, with respect to constraints, $\beta$, on the admissible locations of the various components and the values of the clock and data variables.

The model-checking is performed using backwards reachability analysis together with an efficient constraint-solving technique. Also, UPPAAL adopts onthe-fly generation of the state space in order to avoid explicit construction of the product automaton and the immediately caused memory problems.

The model-checking is based on a partitioning of the (otherwise infinite) statespace into finitely many symbolic states of the form $[\bar{l}, U]$, where $U$ is a simple constraint system (i.e. a conjunction of atomic clock and data constraints ${ }^{2}$ ). The backwards reachability algorithm checks if a symbolic state $\left[\bar{l}_{f}, U_{f}\right]$ is reachable from the initial state $\left[\bar{l}_{0}, U_{0}\right]$, where $U_{0}$ expresses that all clocks and data variables are initialized to 0 .

The algorithm essentially performs a backwards, breadth-first search of the symbolic states. The search is guided and pruned by two buffers: Wait, holding the symbolic states waiting to be explored and Passed holding the symbolic states under exploration and already explored. Initially Passed is empty and Wait holds the single symbolic state $\left[\bar{l}_{f}, U_{f}\right]$. The algorithm then repeats the following:

1. Pick a state $\left[\bar{m}, U^{\prime}\right]$ from the Wait buffer.

2. Check if $\bar{m}=\bar{l}_{0}$ and $U_{0} \subseteq U^{\prime}$. If this is the case, return the answer yes.

3. If $\bar{m}=\bar{n}$ and $U^{\prime} \subseteq U^{\prime \prime}$, for some $\left[\bar{n}, U^{\prime \prime}\right]$ in the Passed buffer, drop $\left[\bar{m}, U^{\prime}\right]$ and go to step 1 . Otherwise save $\left[\bar{m}, U^{\prime}\right]$ in the Passed buffer.

4. Find all symbolic states $[\bar{o}, Z]$ that lead to $\left[\bar{m}, U^{\prime}\right]$ in one step and store them in the Wait buffer.

5. If the Wait buffer is not empty go to step 1, otherwise return the answer no.

${ }^{2}$ Simple constraint systems are also know under the term zone. 
We will not treat the algorithm in more detail here, but refer the reader to to [YPD94, BL96].

Despite its on-the-fly examination of the symbolic state space the above algorithm is bound to run into space problems for sufficiently large systems witnessed by an explosion in the size of the Passed buffer, which is used to record the states already visited in order to enable pruning of redundant examinations (in 3) and eventually ensure termination. The key question is how to limit the growth of this buffer? When using committed locations to ensure atomicity of finite transition sequences of one component (as in modelling broadcast) it obviously suffices to save the symbolic state at the beginning of the sequence. Hence, our proposed solution is simply not to save symbolic states in the Passed buffer which involves committed locations. We therefore modify step 3 of the algorithm in the following way:

3'. a. If committed $(\bar{m})$ go directly to step 4 .

b. If $\bar{m}=\bar{n}$ and $U^{\prime} \subseteq U^{\prime \prime}$, for some $\left[\bar{n}, U^{\prime \prime}\right]$ in the Passed buffer, drop $\left[\bar{m}, U^{\prime}\right]$ and go to step 1 .

c. If neither of the above steps are applicable, save $\left[\bar{m}, U^{\prime}\right]$ in the Passed buffer.

\section{The Audio Control Protocol with Bus Collision}

In this section an informal introduction to the audio protocol with bus collision is given. The audio control protocol is a bus protocol, all messages are received by all components on the bus. If a component receives a message not addressed to it, the message is just ignored. Philips allows up to 10 components.

Messages are transmitted using Manchester encoding. Time is divided into bit-slots of equal length, a bit " 1 " is transmitted by an up-going edge halfway a bit-slot, a bit " 0 " by a down-going edge halfway a bit-slot. If the same bit is transmitted twice in a row the voltage changes at the end of the first bit-slot. Note that only a single wire is used to connect the components, no extra clock wire is needed. This is one of the properties that makes it a nice (read cheap) protocol.

The protocol has to cope with some problems: (a) The sender and the receiver must agree on the beginning of the first bit-slot, (b) the length of the message is not known in advance by the receiver, (c) the down-going edges are not detected by the receiver. To resolve these problems the following is required: Messages must start with a bit " 1 " and messages must end with a down-going edge. This ensures that the voltage on the wire is low between messages. Furthermore the senders must respect a 'radio silence' between the end of a message and the beginning of the next one. This radio silence marks the end of a message and the receiver knows that the next up-going edge is the first edge of a new message. It is (almost) possible to decode a Manchester encoded message by only looking to the up-going messages (problem c) only the last zero bit of a message can not be detected (consider messages "10" and " 1 "). To resolve this it is required that all messages are of odd length. 
It is possible that two or more components start transmitting at the same time. The behavior of the electric circuit is such that the voltage on the wire will be high as long as one of the senders pulls it high. In other words: The wire implements the or-function. This makes it possible for a sender to notice that someone else is also transmitting. If the wire is high while it is transmitting a low, a sender can detect a bus collision. This collision detection happens at certain points in time. Just before each up-going transition, and at one and three quarters of a bit-slot after a down going edge (if it is still transmitting a low). When a sender detects a collision it will stop transmitting and will try to retransmit its message later.

If two messages are transmitted at the same time and one is a prefix of the other, the receiver will not notice the prefix message. To ensure collision detection it is not allowed that a message is a prefix of an other message in transit. In the Philips environment this restriction is met by embedding the source address in each message (and assigning each component a unique source address).

In Figure 2 an example is depicted. Two senders start transmitting at exactly the same time. Because two lines on top of each other is hard to distinguish from one line, they are shifted slightly. The thick sender starts transmitting "11..." and the other "101...". At the end of the first bit-slot the thick sender does a down, to prepare for the next up-going edge. But one quarter after this down it detects a collision and stops transmitting. The thin sender did not notice the other and continues transmitting. Note that the receiver will decode the message of the thin sender correctly.

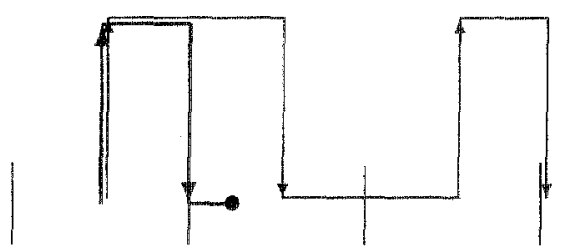

Fig. 2. an example

The protocol has to cope with one more thing: timing uncertainty. Because perfect clocks do not exist in the physical world and because the protocol is implemented on a processor that also has to execute a number of other time critical tasks, a quite large timing uncertainty is allowed. A bit-slot is $888 \mathrm{mi}$ croseconds, so the ideal time between two edges is 888 or 444 microseconds. On the generation of edges a timing uncertainty of $\pm 5 \%$ is allowed. That is: between 844 and 932 for one bit-slot and between 422 and 466 for half a bit-slot. The collision detection just before an up-going edge and the actual generation of this up-going edge must be at most 20 microseconds. The timing uncertainty on the collision detection on one and three quarters after the generation of a down-going edge is \pm 22 microseconds. Also the receiver has a timing uncertainty of $\pm 5 \%$. And, to complete the timing information, the distance between the end of one 


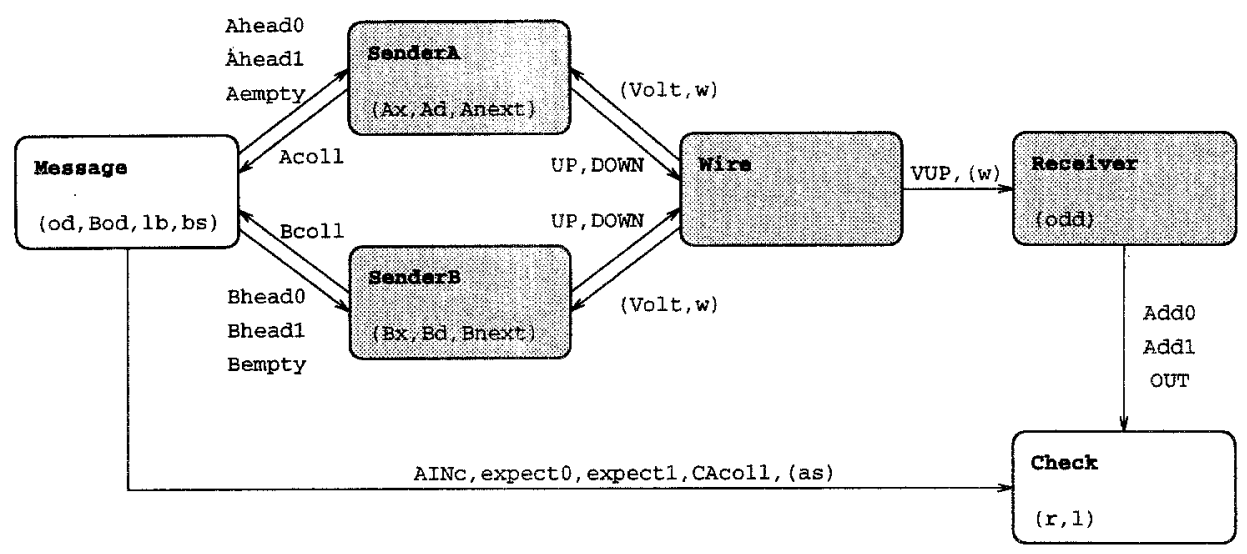

Fig. 3. Philips Audio-Control Protocol with Bus Collision.

message and the beginning of the next must be at least 8000 microseconds ( 8 milliseconds).

\section{A Formal Model of the Protocol}

To analyze the behavior of the protocol we model the system as a network of six timed automata. The network consists of two parts: a core part and a testing environment. The core part models the components of the protocol to be implemented: two senders, a wire and a receiver. The testing environment, consisting of a message generator and an output checker, is used to model assumptions about the environment of the protocol and for testing the behavior of the core part. Figure 3 shows a flow-graph of the network where nodes represent timed automata and edges represent synchronization channels or shared variables (enclosed within parenthesis).

The general idea of the specification is as follows. The automaton Message generates messages for both senders, and also informs the Check automaton on the bits it generated for SenderA. The senders transmit the messages via the wire to the receiver. The receiver communicates the bits it decoded to the checker. Thus the Check automaton is able to compare the bits generated by Message and the bits received by Receiver. If this matches the protocol is correct.

The senders A and B are, modulo renaming (all A's in identifiers to B's), exactly the same. Because of this symmetry, it is enough to check that the messages transmitted by sender $A$ are received correctly. We will proceed with a short description of each automaton. The definition of these uses a number of constants that are declared in Figure 4.

The Senders. SenderA is depicted in Figure 5. It takes input actions Ahead0?, Ahead1? and Aempty?. The output actions UP! and DOWN! will be the Manchester encoding of the message. The clock $A x$ is used to measure the time between 
UP! and DOWN! actions. The idea behind the specification (taken from [DY95]) is that the sender changes location each half of a bit-slot. The locations HS (wire is high in second half of bit-slot) and HF (high in first half of bit-slot) refer to this idea. Extra locations are needed because of the collision detection.

The clock Ad is used to measure the time elapsed between the detection just before UP! action and the corresponding UP! action. Furthermore the time elapsed since the last DOWN! action is measured. The system is in the locations ar_Qfirst and ar_Qlast when the next thing to do is the collision test at one or three quarters of a bit-slot. When Volt is greater than zero, at that moment, the sender detects a collision, stops transmitting and returns to the idle location. The clock $w$ is used to ensure the 'radio silence' between messages. This variable is checked on the transition from idle to ar_first_up.

The Wire. This small automaton keeps track of the voltage on the wire and generates VUP! actions when appropriate, that is when a UP? action is received when the voltage is low.

The Receiver. Receiver (Figure 6) decodes the bit sequence using the up-going (modeled as VUP?) changes of the wire. Decoded bits are signaled to the environment using output actions Add0!, Add1! and OUT! (OUT! is used for signaling the end of a decoded message). The decoding algorithm of the receiver is a direct translation of the algorithm in the Philips documentation of the protocol. In the automaton each VUP? transition is followed by a transition modeling the decoding. This decoding happens 'at once' therefore these intermediate locations are modeled as committed locations. The automaton has two important locations, $L 1$ and $L 0$. When the last received bit is a bit " 1 " the receiver is in location $L 1$, after receiving a bit " 0 " it will be in location LO. The error location is entered when a VUP? is received much to early. In the complete specification the error location is not reachable, see Section 6 . The receiver keeps track of the parity of the received message using the integer variable odd. When the last received bit is a bit " 1 " and the message is even, a bit " 0 " is added to make the complete message of odd length.

The Message Generator. The message generator generates messages of odd length for both sender A and B. Furthermore, the messages generated for sender A, are communicated to the checker. When a collision is detected by sender $A$ this is communicated to the message generator via Acoll?. The message generator will communicate this on his turn to the checker via CAcoll!. Generating messages of odd length is quite simple. The only problem is that it is not allowed that a message for one sender is a prefix of the message for the other sender. To be more precise: If only one sender is transmitting there is no prefix restriction. Only when the two senders start transmitting at the same time, it is not allowed that one sender transmits a prefix of the message transmitted by the other. As mentioned before the reason for this restriction is that the prefix message is not received by the receiver and it is possible that the senders do not notice the collision. In other words: The prefix message can be lost.

The Checker. This automaton keeps track of the bits 'in transit', that is the 
bits that are generated by the message generator but not yet decoded by the receiver. Whenever a bit is decoded or the end of the message is detected not conform the generated message the checker enters an error location. Furthermore when sender A detects a collision the checker returns to its initial location.

\section{Verification in UPPAAL}

In this section we verify correctness of the protocol described in previous sections. Recall, that the system is modelled as a network of the six timed automata: Message SenderA, SenderB, Wire, Receiver and Check, and that properties are specified as logical formulas.

The Correctness Criteria. The correct behaviour of the protocol is ensured whenever the control of the automaton Check is in location a or start. If an incorrect behaviour is detected the Check-automaton enters the error-location, consequently property (1) requires that the Check-automaton is always in location start or a:

$$
\forall \square(\text { Check.start } \vee \text { Check.a) }
$$

For the property to be satisfied it is required that the bit sequence received by the Receiver matches the bit sequence sent by SenderA. Furthermore, it is also required that the entire bit sequence is received by Receiver (and communicated to the Check-automaton). This is ensured since the error-location of the Checkautomaton is reachable if the end of a bit sequence is signalled by Receiver (i.e. OUT!) when unmatched bits exists in the Check-automaton.

If the Receiver-automaton observes changes of the wire too early in location L1 or L0 control is changed to location error. It is imaginable that error recovery can be implemented from this location. However, if the other components of the protocol conform to the specification this location should not be reachable, thus property (2) requires that the error-location in Receiver is never reachable.

$$
\forall \square \neg \text { Receiver.error }
$$

Incorrectness. Unfortunately the protocol described in this paper is not the protocol that Philips has implemented. The original sender checked less often for bus collisions. The 'just before the up going edge' collision detection was only performed before the first up. (In our modelling this corresponds to modifying SenderA and SenderB in the following way: delete the outgoing transitions of location ar_Qlast_ok and use the outgoing transitions of location ar_up_ok instead.) This version is incorrect. In general the problem is that if both senders are transmitting and one is slow and the other fast, the distance can cumulate to a high value and this can confuse the receiver. UPPAAL generated a counter example trace.

Although this problem was known by Philips is it interesting to see how powerful the diagnostic traces can be. It enables us not only to find mistakes in the model of a protocol, but also to find design mistakes in real life protocols. 
The Verification Results. UPPAAL successfully verifies the correctness properties (1) and (2) for an error tolerance of $5 \%$ on the timing. Recall that SenderA and Sender $B$ are, modulo renaming, exactly the same, implying that the verified properties for SenderA also applies to the symmetric case for SenderB. Property (1) was verified in $7.5 \mathrm{hrs}$ using $527.4 \mathrm{MB}$ of memory, property (2) in 1.32 hrs using $227.9 \mathrm{MB}$ of memory.

The analysis of the incorrect version of the protocol with less collision detection (discussed above) uses UPPAAL's ability to generate diagnostic traces whenever a certain property is not satisfied by the system. The trace, consisting of 46 transitions, was generated in 13.0 min using $290.4 \mathrm{MB}$ of memory. Also, attempts to verify Property (1) for the full protocol with an error tolerance of $6 \%$ on the timing failed. The scenario is similar to the one found by Bosscher et al. in [BPV94] for the one sender protocol.

The properties (above) were verified using the verification algorithm for handling committed locations, described in Section 3 , implemented in a new prototype version of UPPAAL, installed on a SGI ONYX.

\section{Conclusion}

In this paper it is shown to be possible to verify properties of a realistic case study using UPPAAL. The tool is able to verify the correctness properties of the Philips Audio Protocol, that is: the receiver only receives messages that are transmitted. Furthermore the ability of UPPAAL to generate diagnostic traces proved very useful. When writing formal specifications (some) humans tend to make mistakes. These mistakes are much easier to locate using a tool that can generate scenarios. This in contrast with using a tool that only provides Yes/No answers to queries.

We proposed the use of committed locations in UPPAAL specifications. Using these provides a significant efficiency improvement. Furthermore the memory consumption decreases when using committed locations.

Even more important than the efficiency improvement is that committed locations sometimes allow a more natural specification. If a system does a broadcast or multi-way synchronization, this can be modelled much nicer using committed locations. Without committed locations it is not possible in UPPAAL to prohibit other components to perform actions during the broadcast. With committed locations these multi communications can be modelled as a single atomic action.

Another option to model broadcast synchronization is to use another synchronization mechanism than handshake as used in UPPAAL. We prefer the use of committed locations because it is easier to embed in the model and easier to implement. We also think that committed locations and handshake synchronization provide a flexible and expressive model for specifying protocols.

\section{References}

[AD90] R. Alur and D. Dill. Automata for Modelling Real-Time Systems. In Proc. of ICALP'90, LNCS 443, 1990. 
[BL96] Johan Bengtsson and Fredrik Larsson. UPPAAL a Tool for Automatic Verification of Real-time Systems. Master's thesis, Uppsala University, 1996.

[BLL ${ }^{+}$95] Johan Bengtsson, Kim G. Larsen, Fredrik Larsson, Paul Pettersson, and Wang Yi. UPPAAL- a Tool Suite for Automatic Verification of RealTime Systems. In Proc. of the 4th DIMACS Workshop on Verification and Control of Hybrid Systems, 1995. To appear in LNCS, 1996.

[BPV94] D.J.B. Bosscher, I. Polak, and F.W. Vaandrager. Verification of an AudioControl Protocol. In Proc. of FTRTFT'94, LNCS 863, pages 170-192, 1994.

[DY95] C. Daws and S. Yovine. Two examples of verification of multirate timed automata with KRonos. In Proc. of the 16th IEEE Real-Time Systems Symposium, pages 66-75, December 1995.

[Gri94] W.O.D. Griffioen. Analysis of an Audio Control Protocol with Bus Collision. Master's thesis, University of Amsterdam, Programming Research Group, 1994.

[HHWT95] Thomas A. Henzinger, Pei-Hsin Ho, and Howard Wong-Toi. HyTECH: The Next Generation. In Proc. of the 16th IEEE Real-Time Systems Symposium, pages 56-65, December 1995.

[HRP94] N. Halbwachs, P. Raymond, and Y.-E. Proy. Verification of linear hybrid systems by means of convex approximations. In Static Analysis Symposium, LNCS 864, pages 223-237, 1994.

[HWT95] Pei-Hsin Ho and Howard Wong-Toi. Automated Analysis of an Audio Control Protocol. In Proc. of CAV'95, LNCS 939, 1995.

[LPY95] Kim G. Larsen, Paul Pettersson, and Wang Yi. Diagnostic Model-Checking for Real-Time Systems. In Proc. of the 4th DIMACS Workshop on Verification and Control of Hybrid Systems, 1995. To appear in LNCS, 1996.

[YPD94] Wang Yi, Paul Pettersson, and Mats Daniels. Automatic Verification of Real-Time Communicating Systems By Constraint-Solving. In Proc. of the 7th International Conference on Formal Description Techniques, 1994.

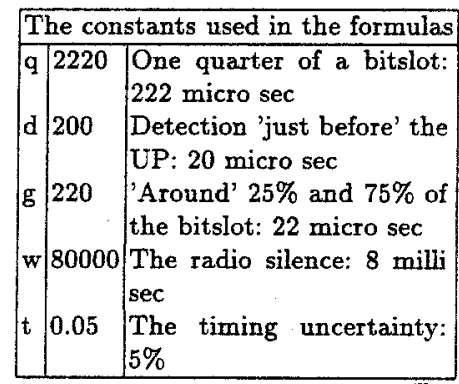

\begin{tabular}{|l|l|l|}
\hline \multicolumn{3}{|l|}{ The constants in the automata } \\
\hline W & $\mathrm{w}$ & 80000 \\
$\mathrm{D}$ & $\mathrm{d}$ & 200 \\
$\mathrm{~A} 1 \min$ & $\mathrm{q}-\mathrm{g}$ & 2000 \\
$\mathrm{~A} 1 \max$ & $\mathrm{q}+\mathrm{g}$ & 2440 \\
$\mathrm{~A} 2 \min$ & $3^{*} \mathrm{q}-\mathrm{g}$ & 6440 \\
$\mathrm{~A} 2 \max$ & $3^{*} \mathrm{q}+\mathrm{g}$ & 6880 \\
$\mathrm{Q} 2$ & $2^{*} \mathrm{q}$ & 4440 \\
$\mathrm{Q} 2 \operatorname{minD}$ & $2^{*} \mathrm{q}^{*}(1-\mathrm{t})-\mathrm{d}$ & 4018 \\
$\mathrm{Q} 2 \min$ & $2^{*} \mathrm{q}^{*}(1-\mathrm{t})$ & 4218 \\
\hline
\end{tabular}

\begin{tabular}{|l|l|l|}
\hline \multicolumn{3}{|c|}{ The constants continued } \\
\hline Q2max & $2^{*} \mathrm{q}^{*}(1+\mathrm{t})$ & 4662 \\
Q3min & $3^{*} \mathrm{q}^{*}(1-\mathrm{t})$ & 6327 \\
$\mathrm{Q} 3 \max$ & $3^{*} \mathrm{q}^{*}(1+\mathrm{t})$ & 6993 \\
Q5min & $5^{*} \mathrm{q}^{*}(1-\mathrm{t})$ & 10545 \\
Q5max & $5^{*} \mathrm{q}^{*}(1+\mathrm{t})$ & 11655 \\
Q7min & $7^{*} \mathrm{q}^{*}(1-\mathrm{t})$ & 14763 \\
Q7max & $7^{*} \mathrm{q}^{*}(1+\mathrm{t})$ & 16317 \\
Q9min & $9^{*} \mathrm{q}^{*}(1-\mathrm{t})$ & 18981 \\
Q9max & $9^{*} \mathrm{q}^{*}(1+\mathrm{t})$ & 20979 \\
\hline
\end{tabular}

Fig. 4. Declaration of Constants. 


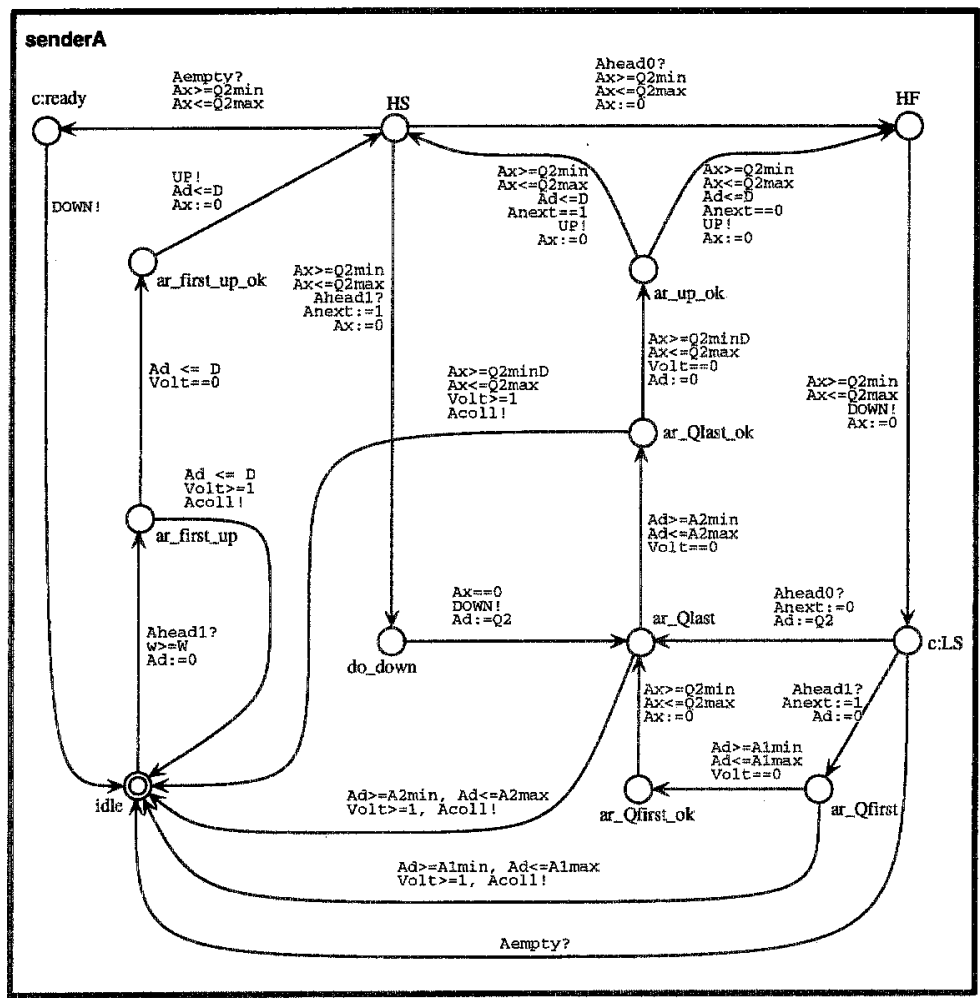

Fig. 5. The SenderA Automaton.

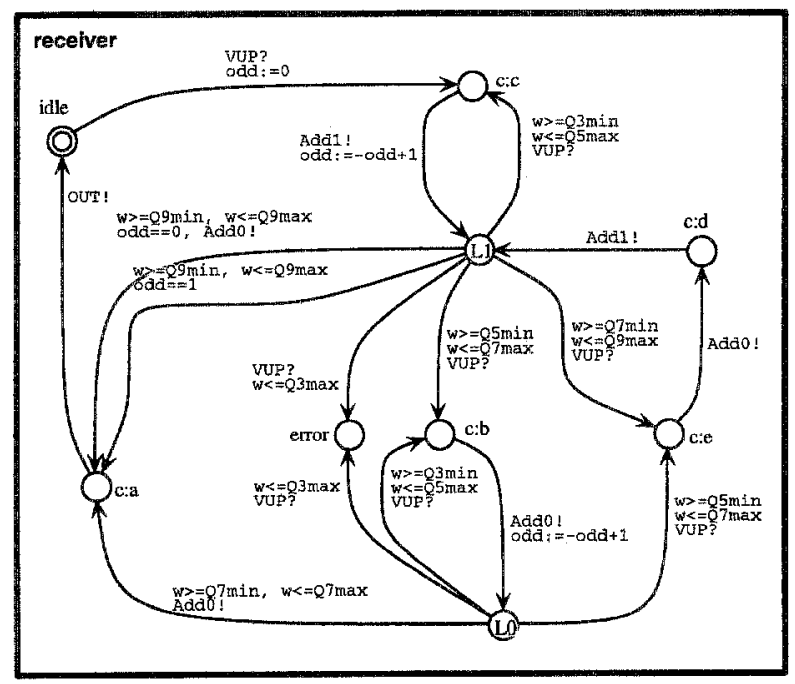

Fig. 6. The Receiver Automaton. 